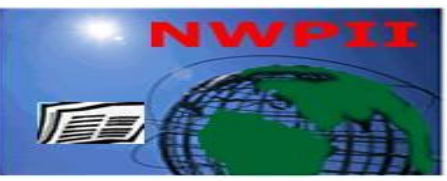

American Journal of Biomedical Sciences

ISSN: 1937-9080

nwpii.com/ajbms

\title{
Calcium Phosphate Scaffold Loaded with Platinum Nanoparticles for Bone Allograft
}

\author{
Kamel Eid $^{1^{*}}$, Amal Eldesouky ${ }^{2}$, Ali Fahmy ${ }^{3}$, Ahmed Shahat $^{4}$, and Reda AbdElaal ${ }^{5}$ \\ ${ }^{1,4,5}$ Department of Chemistry, Faculty of Science, Suez University, Suez, Egypt \\ ${ }^{2}$ Department of Biomedical Engineering, Higher Technology Institute, HTI, $10^{\text {th }}$ of Ramadan City, EGYPT \\ ${ }^{3}$ Applied Research Sector, Egy-Vac/ VACSERA, 22311, (1), EGYPT \\ Corresponding author: \\ Kamel A.M. Eid \\ School of Sciences \& Engineering \\ The American University In Cairo \\ AUC Avenue, SSE \# 1184 \\ P.O. Box 74 \\ New Cairo, Egypt 11835 \\ Fax: +20227957565 \\ E-mail:kamelam@aucegypt.edu
}

Received: 6 June 2013; | Revised: 2 November 2013; | Accepted: 23 November 2013

\section{Abstract}

Inflammations are a predicament issue that leads to orthopaedic hard implants failure. Thereby, calcium phosphate platinum scaffolds possess anti-inflammatory properties, as powerful tools for successful bone regeneration. The effects of the platinum nanoparticles (PtNPs) on the scaffold's degradation and proliferation and attachment were evaluated. The scaffolds degradation rates ranged between $50-75 \%$ and 80-95\% for Scaffold with and without PtNPs respectively. Moreover, the cells proliferation and attachment on CPs-PtNPs scaffold were superior to CPs scaffold. The results warranted that, the synthesized scaffolds exhibit good biocompatibility and in vitro biodegradation, and also, it could be a used as a substrate for PtNPs delivery.

Keywords: Platinum Nanoparticles, Tissue Engineering, Bone Grafting, Calcium Phosphate.

\section{Introduction}

Tissue engineering is a fast growing field that provides unique alternative solutions for organ transplants, and thus offers dramatic potential improvement in human health( $(1)$. Autologous and allograft techniques for bone grafting are the most common choice for filling large bone defects (2-3). However, these approaches have their drawbacks, such as the need for second surgery at the donor site, limited 
quantity and shape of available bone, and resorption of the bone graft(4-5). A range of porous materials, such as ceramics, polymers and metals have been developed in the field of bone grafts and scaffolds for tissue engineering(2-10). Some of the most favourable bioactive ceramic materials that were used in bone grafting and tissue engineering are calcium phosphate due to their exceptional physical and chemical properties $(2,4-5)$. One of the most unique properties for such materials are the mechanical, biological and protein absorption of composite used for scaffold synthesis(2, 4, 6). Consequently, they have been extensively used in biomedical implants for bone regeneration, such as coating for metallic implants, bone grafts and composites for middle bone implants $(4,7)$. In addition to their biocompatibility and biodegradability, the osteoconductive properties of these composites make them ideal materials for bone regeneration(7-8). However, their poor mechanical properties, in particular their toughness, is a significant problem Successful incorporation of calcium phosphate with polymers has been achieved for the fabrication of scaffolds that have good porosity, degradability and protein absorption, while accomplishing acceptable mechanical properties in term of toughness (7-8). These scaffolds were subsequently used as an alternative autologous bone graft for tissue engineering(8-9). Generally, tissue engineering scaffolds should have suitable pore area micro-pore size, degradability and good mechanical properties (10). Thereby, designing a scaffold material from ceramics with other materials such as metals or polymers may lead to good simulation of the structure and properties of tissues to be replaced, and offers a tremendous potential for solving these problems (9-10).

Noble metal nanostructures are of considerable interest because of their unique properties and have been successfully introduced in many applications such as, antimicrobial applications, tissue engineering, and diagnostics (11-16). Therefore, noble metal nanostructures have been synthesized in many forms, such as wires, and flowers and have been successfully used in many applications such as medical (1116). Platinum nanostructures are of particular interest for many applications, including sensing, and medical applications such as potential medicinal substance for oxidative stress diseases with suppressed mitochondrial complex (18-21) . Pt NPs show antioxidant properties that herald a promising future for the treatment of oxidative-stress-related conditions, such as neurodegenerative disorders, and including Alzheimer's diseases (18-21). Additionally, PtNPs have been shown to protect cells from oxidation-induced inflammation which inhibits pulmonary inflammation and induced bone loss by decreasing osteoclastogenesis(18-22). There are many versatile methods for biomaterials scaffolds fabrication such as freeze dryer, solvent casting, laser, and gas foaming.(23-27 This method yield wide range of scaffolds varies in both chemical and physical properties. This methods result scaffolds with highly load bearing properties but with lack of degradations. Herein, we have focused on the synthesis of biodegradable and safe calcium phosphate scaffold loaded with Pt NPs.To the best of our knowledge this is the first report on the combinations between PtNPs and CPS as scaffolds for bone allograft. Also, this is the first report on the effect of Pt NPs on the scaffolds degradation rate.

\section{Materials and methods}

\subsection{Materials}

Hydrogen hexachloro platinate hexahydrate $\left(\mathrm{H}_{2} \mathrm{PtCl}_{6}{ }_{6} \mathrm{H}_{2} \mathrm{O} ; 99.99 \%\right)$, tri-sodium citrate $\left(\mathrm{Na}_{3} \mathrm{C}_{6} \mathrm{H}_{5} \mathrm{O}_{7}, 98 \%\right)$, Dulbecco's Modification of Eagles Med (DMEM) ,glutamine, penicillin, streptomycin and (3-(4,5-Dimethylthiazol-2-yl)2,5-diphenyltetrazolium bromide, polyvinyl alcohol, Diammonium hydrogen phosphate $\left(\mathrm{NH}_{4}\right)_{2} \mathrm{PO}_{4}$, and Calcium nitrate $\mathrm{Ca}\left(\mathrm{NO}_{3}\right)_{2}$ were purchased from Sigma-Aldrich Chime $\mathrm{GmbH}$ (Munich, Germany). Ammonium Solution $\mathrm{NH}_{4} \mathrm{OH}$ (35\%), Poly Carbonate Filter paper, Hydrogen chloride $\mathrm{HCL}$ and Nitric acid $\mathrm{HNO}_{3}$ were purchased from El-gomhouria Co, (Cairo, Egypt). Double deionized water (DDI) was prepared using a Milli-Q ${ }^{\mathrm{TM}}$ system (Direct-Q 3, Model ZRQSOPOWW, Millipore Corporation, Billerica, MA) with a resistivity of $18 \mathrm{MÜcm}$. 


\subsection{Synthesis of CPs-PtNPs Scaffold}

The calcium phosphate and PtNPs were synthesized in our lap. In Concise, calcium phosphate platinum nanoparticles CPs-PtNPs scaffolds were fabricated by heat sintering. Briefly, calcium phosphate with different concentrations (namely: 20, 30, and $40 \mathrm{wt} \%$ ) were dissolved in DDI. The powder was added to poly vinyl alcohol solution (2 wt \%) to create ceramic slurries. Next, Pt NPs solutions of $10 \mathrm{wt} \%$ were added to the slurries mixture with slow stirring and heating to complete mixing. As a cross-linker, a solution of $2 \mathrm{ml}$ of $2.5 \%$ glutaraldehyde was added drop wise. Finally the slurries were cast into a mould and dried at 100 ${ }^{\circ} \mathrm{C}$ for 24 hours and then sintered at $400{ }^{\circ} \mathrm{C}$ by incrementing $10{ }^{\circ} \mathrm{C}$ per minute for 8 hours. The moulds were allowed to cool at a rate of $10{ }^{\circ} \mathrm{C}$ per minute until they reached room temperature.
The calcium phosphate scaffolds were synthesized as described above but free of PtNPs.

\subsection{Cells proliferation and attachment}

Scaffolds of size $14 \times 10 \mathrm{~mm}$ were seeded with culture medium human osteoblast cells for a period of 21 days. The scaffolds were submerged in $70 \%$ ethanol for sterility and autoclaved for two hours at $130^{\circ} \mathrm{C}$. Then, they were washed with phosphate buffered saline (PBS - pH: 7.4) and soaked in $5 \mathrm{ml}$ of culture medium for one hour. Human osteoblast cells were grown in 24 well plates at a density of $5 \times 10^{6}$ cells $/ \mathrm{ml}$ in Medium (DMEM) containing fetal bovine serum $(10 \% \mathrm{~V} / \mathrm{V})$, glutamine $(2 \mathrm{mM})$, penicillin $(100$ $\mathrm{U} / \mathrm{mL})$ and streptomycin $(1 \mu \mathrm{g} / \mathrm{mL})$, and incubated in $5 \% \mathrm{CO}_{2}$ at $37{ }^{\circ} \mathrm{C}$. The culture mediums were changed every 48 hours for a period of 14 and 21 days.

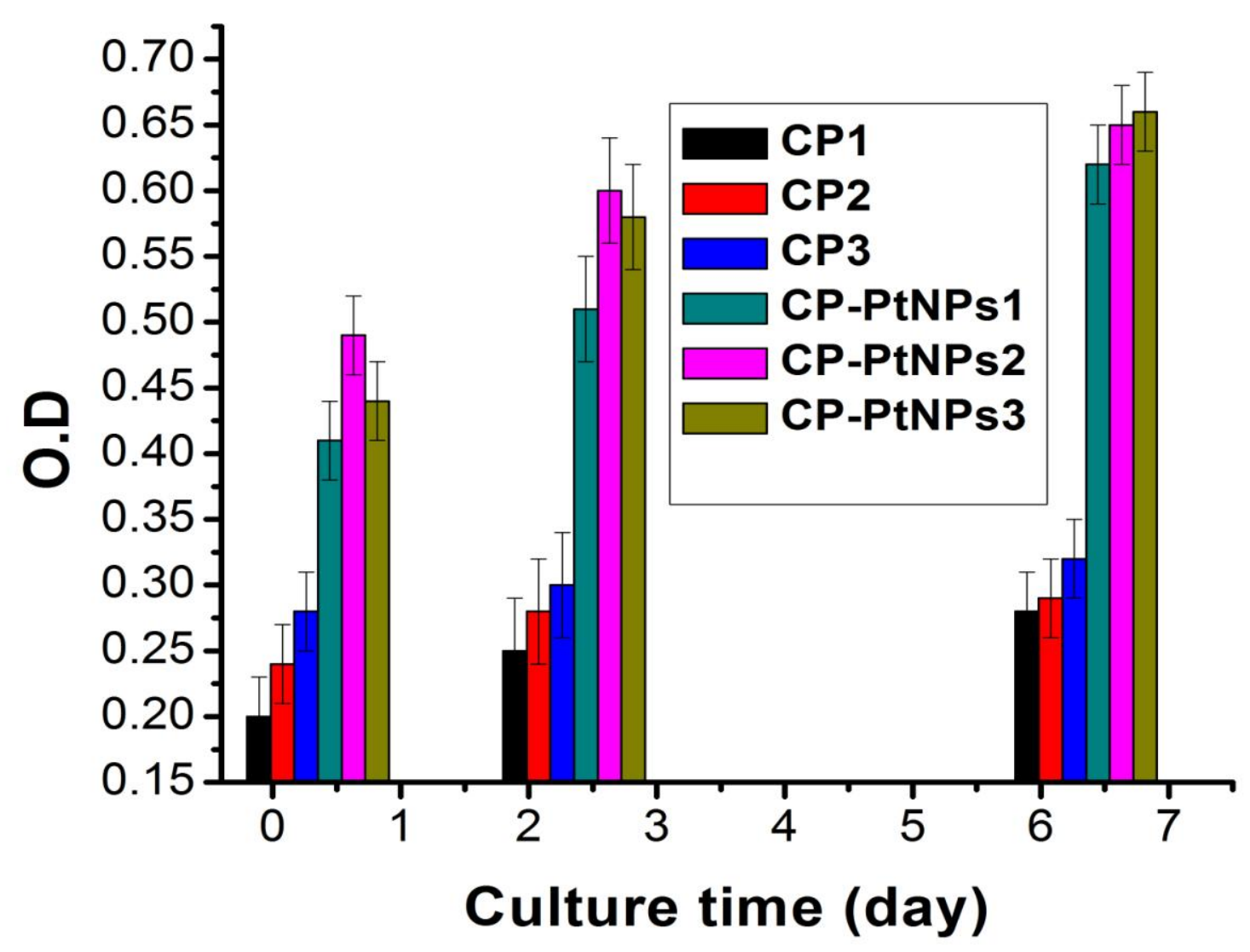

Figure 4. Cells proliferation and attachment on CP 1-3 that have 20, 30, and 40wt $\%$ of CPs powder while, CPs-PtNPs scaffold 1-3 have 20, 30, and 40wt\% of CPs powder with $10 \%$ wt of Pt. 


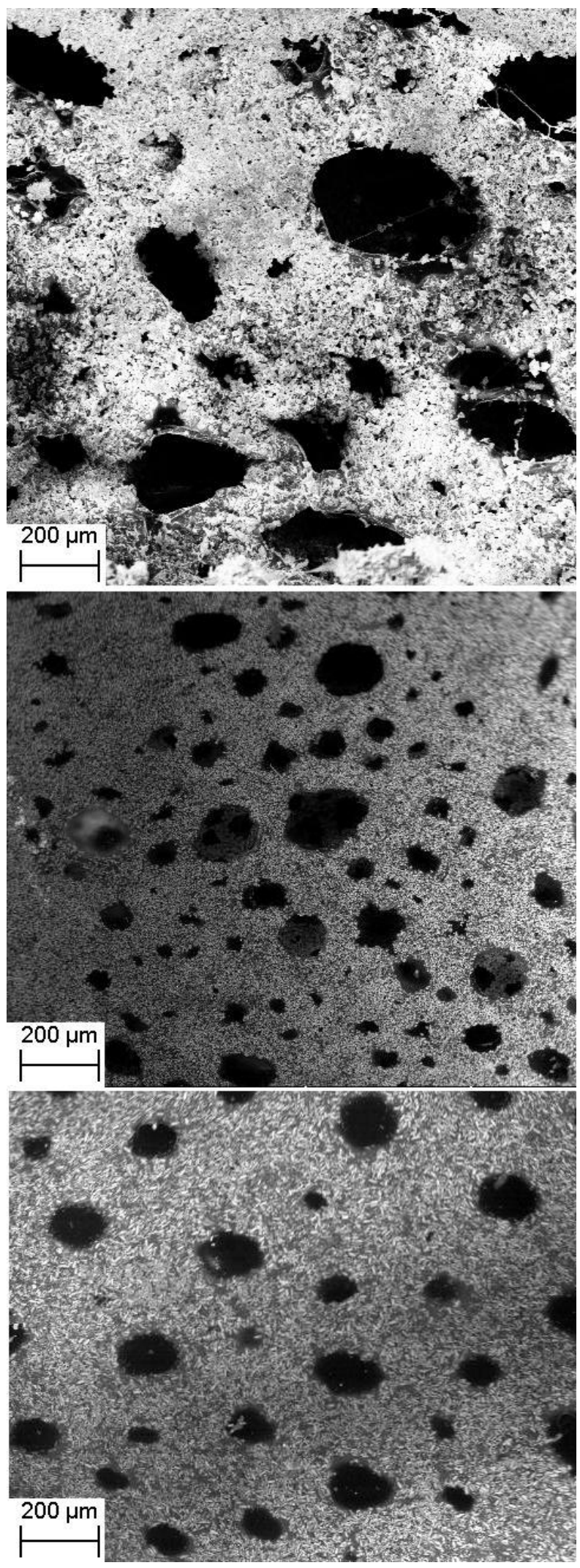

Figure 1. Ceramic scaffold that were synthesized upon using of 20, 30 and $40 \mathrm{wt} \%$ of CPs nanoparticles without PtNPs [A-C] respectively.
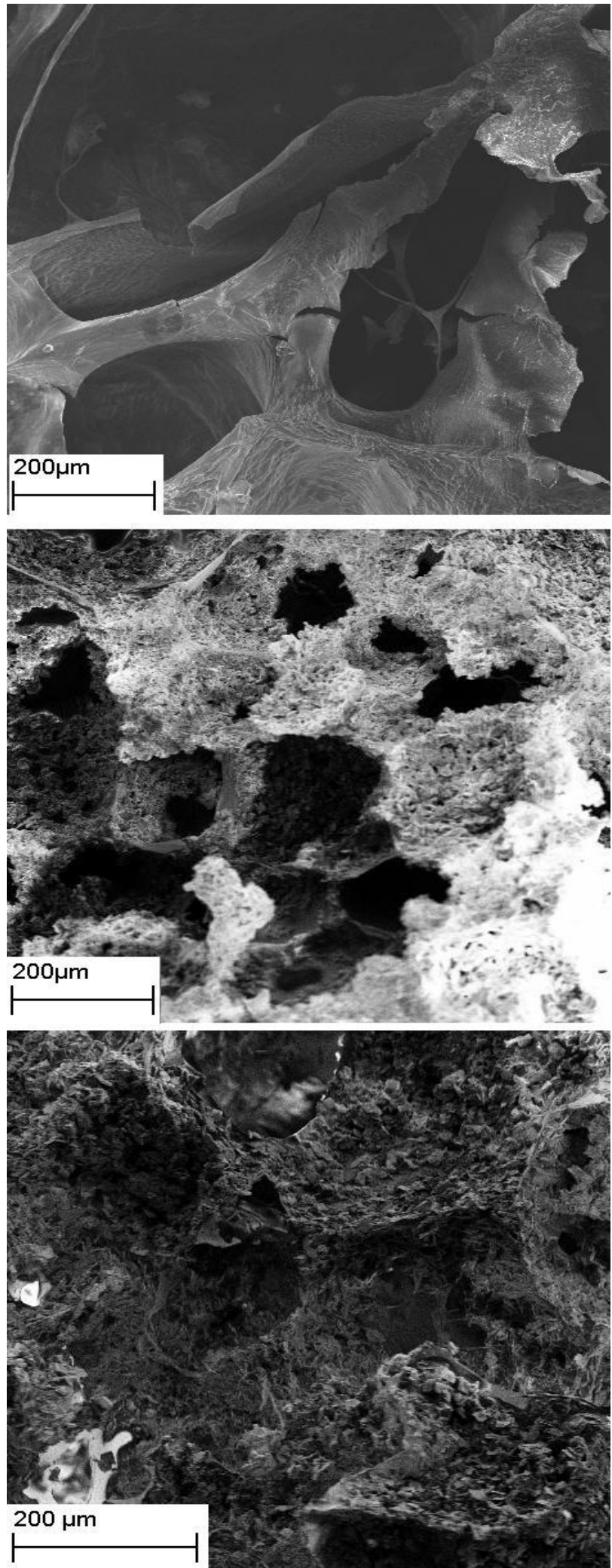

Figure 2. Ceramic scaffolds that were synthesized upon using of 20,30 and $40 \mathrm{wt} \%$ of CPs nanoparticles without PtNPs [A-C] respectively. 


\subsection{Scaffold Morphology}

The Micro-structure of the synthesized scaffolds were studied using field emission scanning electron microscope (SEM, LEO SUPRA 55; Carl Zeiss AG, Oberkochen, Germany) at an accelerating voltage of $8-12 \mathrm{kV}$, with magnification in the range of 50-400 Kx. The scaffold samples were mounted on an SEM grid and imaged as it is without sputter coating. The micro-structures were selected randomly from different visions through imaging process.

\subsection{In vitro Degradation Test of the scaffold}

In vitro degradation of the scaffolds were investigated by cutting the scaffold samples to size $12 \times 5 \mathrm{~mm}$ and soaking them in PBS 7.4 at $37^{\circ} \mathrm{C}$ in shaking water bath for 6 hours, 1, 3, 7, 14 , and 28 days. The solutions were refreshed every day and were measured using changes in dry weight after incubation for a specified time period. For such tests, the specimens were removed, rinsed in distilled water, and dried in a vacuum oven for 1 day at $120^{\circ} \mathrm{C}$. All the values presented are the average of three specimens. The percentage of weight loss was computed using the following equation:

Weight loss $=\frac{\mathrm{W}_{\mathrm{t}}-\mathrm{W}_{0}}{\mathrm{~W}_{0}} \mathrm{X} 100$

Where $\mathrm{W}_{0}$ is the starting dry weight and $\mathrm{W}_{\mathrm{t}}$ is the dry weight at time $t$.

\section{Results and discussion}

\subsection{Scaffolds morphology and micro-structures}

The size and morphology of the CPs-PtNPs were studied using SEM, and the particle size. The CPs scaffolds poses a moderate porosity but with lake of interconnectivity. Briefly the scaffold sample synthesized by using of $20 \mathrm{wt} \%$ of CPs powder shows the best porosity ranged between $30-35 \%$ with pore size ranging between $100-400 \mu \mathrm{m}$. Also the pores have canal like. The porosity for scaffolds samples synthesized upon usage of $30,40 \mathrm{wt} \%$ displayed porosity in the range of $20-10 \%$ with pore size ranged between
100-300 $\mu \mathrm{m}$. These results demonstrated that, the porosity decreases with increasing of CPs powder concentration with lake of interconnected pores.

The fabricated CPs-PtNPs scaffold displayed a highly porous and interconnected open-pore structure with pore size distribution ranging between 100-800 $\mu \mathrm{m}$, as shown in Fig.2 A-F. Briefly, Fig.2 A-C presents the scaffolds samples that were synthesized by using 20,30 and $40 \mathrm{wt} \%$ of CPs nanoparticles with $10 \%$ of Pt NPs. Other scaffolds were synthesized with 20,30, and $40 \%$ Pt NPs with $40 \mathrm{wt} \%$ of CPs nanoparticles to manipulate and study the effects of varying PtNPs on the scaffolds morphology as is demonstrated in Fig.2( D-I). The observed final porous structures of the scaffold are clearly a function of CPs-PtNPs ratios and PtNPs concentration. Thus, it may be deduced that increasing CPs-PtNPs and/or PtNPs concentrations increases both of the porosity and interconnectivity of the scaffolds. Therefore, the scaffolds open and form interconnected pores due to the presence of PtNPs with CPs powder that may act as non-solvent that lead to enhanced porosity and interconnectivity. The Interconnected pore structure is important to bone cell-growth, tissue regeneration, and interface support. Furthermore, the micro-porous structure is beneficial to capillary growth, nutrient transport, and biological properties of the implant (1-7). Several groups reported that, the porous surface improves the mechanical interlocking between the implant and the surrounding natural bone, providing greater mechanical stability at the critical interface (7-10, 22-25).

\subsection{In vitro Degradation Test}

To support the differentiation and proliferation of cells, a scaffold should be biodegradable and bioactive. The degradability of the scaffold samples were monitored at 3, 5, 10, 15, and 25 days in culture, as shown in Fig. 4. Obviously, the degradation rate for calcium phosphate scaffolds sample 1-3 synthesized upon usage of $20,30,40 \mathrm{wt} \%$ of CPs, increased regularly up to day 25 and ranged between 10 $90 \%$. The degradation rates increased hastily at the first 15 days and ranged between $8-78 \%$. 
Their small difference between degradation rates for samples. Also, both of samples 1 and 2 degrade correspondingly. Apparently, the degradation rates of CPs-PtNPs scaffolds sample land 2, increased gradually and ranged between $8-70 \%$. Briefly, the degradation rates were faster through the first 15 days and whereas it decreased at day 15 ranging between $10-60 \%$, followed by a steady increase in the rate degradation up till day 30 , up till $70 \%$. While, the decrease in degradation rate at day 20 and 25 for the scaffolds sample 3 and 5 may due to the depositing and partially saturating the SBF solution by CPs-PtNPs powder or may due to the unknown behaviours of nanoparticles(11-16,18-
25). These results indicate that Pt NPs delayed the degradation rates of the scaffolds via decreasing the solubility of the scaffold. Rather than relying on the biodegradable scaffold alone, the enhanced mechanical and biological properties of Pt NPs loaded scaffold will definitely be useful when the portion of bone to regenerate is large.

Furthermore, the results clearly revealed that, the PtNPs could induce powder CPs particles to deposit in SBF, and thus has a better bioactivity in vitro, compared to the ceramic scaffolds.
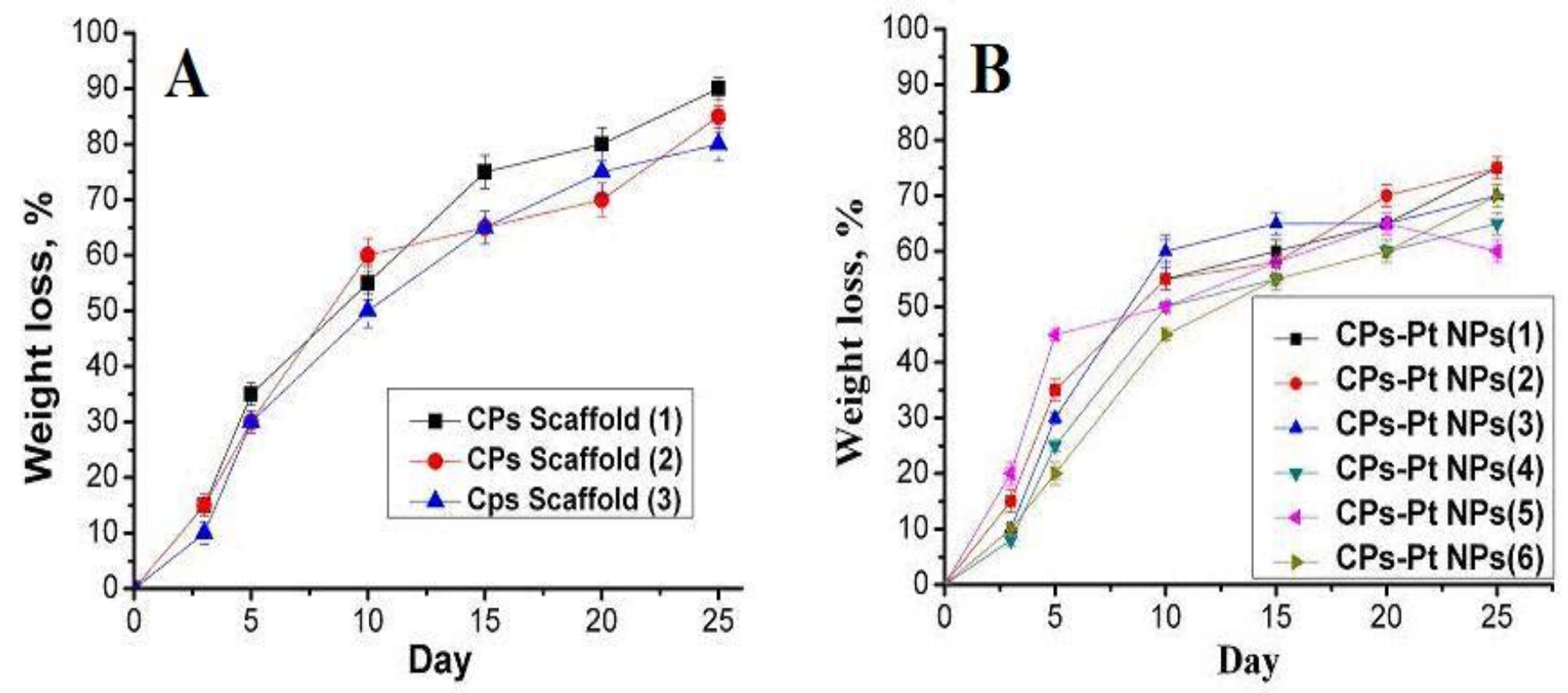

Figure 3. A The degradation rate for calcium phosphate scaffolds sample 1-3 using 20, 30,40wt $\%$ of CPs. While B is the Effect of platinum on degradation rate: Scaffolds using of 20,30,40wt \% of CPs with $10 \%$ of PtNPs sample [1-3 ] and $20,30,40 \%$ of PtNPs with $40 \mathrm{wt} \%$ CPs sample [4-6] on day 3-25.

\subsection{Cell proliferation and attachment}

Proliferation and attachment of Human osteoblast cells cultured on the CPs and CPPtNPs scaffolds were assessed using the MTT assay. Optical density (O.D.) absorbance values were measured by micro-plat reader at day $(1,3$, and 7) which it is accurate and fast methods for an indication of cell proliferation on various biomaterials. Figure 4 reveals that O.D. values for all scaffold samples increased gradually from day 1 to day 7. Also, CPs-PtNPs were significantly higher than those of CPs scaffolds at 7 days. There is a significant difference between CPs and CPs-PtNPs samples. Also, the cells proliferation on CPs-PtNPs 1-3 that have $10 \%$ of PtNPs were higher than CPs scaffold free of PtNPs. These results clearly indicated that, cell growth and proliferation on CPs-PtNPs was superior to $\mathrm{CP}$, which suggesting that CP-PtNPs 
scaffolds assist cell growth and could promote cell proliferation.

\section{Conclusions}

Calcium phosphate scaffold have successfully achieved bioactivity, degradability, and suitable mechanical properties compared to conventional polymeric scaffolds. Herein, we fabricated calcium phosphate platinum scaffolds with good degradability properties. The effects of varying $\mathrm{Pt}$ NPs on the scaffold morphology degradability and cells viability have been studied. The Pt NPs have unambiguous effects on the degradation rates. The in vitro degradation rates of calcium phosphate platinum scaffolds ranged between $8-70 \%$ after 25 days of immersing in SBF. The cells proliferation and attachment on CPs-PtNPs scaffold were superior to CPs scaffold also; the results suggested that the calcium phosphate platinum scaffold's exhibits good biocompatibility and in vitro biodegradability. To the best of our knowledge, platinum nanoparticles have not been incorporated previously with calcium phosphate for fabrication of scaffolds for bone allograft.

\section{References}

1. 1. Ma, P. X. (2004) Scaffolds for tissue fabrication, Materials today 7, 30-40. DOI: 10.1016/S1369-7021(04)00233-0

2. Moore, W. R., Graves, S. E., and Bain, G. I. (2003) Synthetic bone graft substitutes, ANZ journal of surgery 71, 354-361. DOI: 10.1046/j.1440-1622.2001.02128.x

3. Benjamin F. Ricciardi, Mathias P. Bostrom (2013) Bone graft substitutes: Claims and credibility Seminars in Arthroplasty, 24,2, 119-123. DOI: 10.1053/j.sart.2013.07.002

4. Laurencin, C., Khan, Y., and El-Amin, S. F. (2006) Bone graft substitutes, Expert review of medical devices 3, 49-57. DOI: 10.1586/17434440.3.1.49

5. Finkemeier, C. G. (2002) Bone-grafting and bone-graft substitutes, The Journal of Bone \& Joint Surgery 84, 454-464.
6. Hing, K. A. (2005) Bioceramic bone graft substitutes: influence of porosity and chemistry, International journal of applied ceramic technology 2, 184-199. DOI: 10.1111/j.1744-7402.2005.02020.x

7. Ambard, A. J., and Mueninghoff, L. (2006) Calcium phosphate cement: review of mechanical and biological properties, Journal of Prosthodontics 15, 321-328. DOI: 10.1111/j.1532-849X.2006.00129.X

8. Ignjatovic, N., Ninkov, P., Ajdukovic, Z., Vasiljevic-Radovic, D., and Uskokovic, D. (2007) Biphasic calcium phosphate coated with poly-d, 1-lactide-co-glycolide biomaterial as a bone substitute, Journal of the european ceramic society 27, 1589-1594. DOI: 10.1016/j.jeurceramsoc.2006.04.104

9. Epple, M., Ganesan, K., Heumann, R., Klesing, J., Kovtun, A., Neumann, S., and Sokolova, V. (2010) Application of calcium phosphate nanoparticles in biomedicine, Journal of Materials Chemistry 20, 18-23. DOI: $10.1039 / \mathrm{B} 910885 \mathrm{H}$

10. El-Ghannam, A. (2005) Bone reconstruction: from bioceramics to tissue engineering, Expert review of medical devices 2, 87-101. doi:10.1586/17434440.2.1.8

11. Aly, A. F., Eldesouky, A. S., and Eid, K. A. Evaluation, Characterization and Cell Viability of Ceramic Scaffold and Nano-gold loaded Ceramic Scaffold for Bone Tissue Engineering, Cell 4, 316-326.

12. Eid, K. A., Salem, H. F., Zikry, A. A., ElSayed, A. F., and Sharaf, M. A. (2011) Antifungal Effects of Colloidally Stabilized Gold Nanoparticles: Screening by Microplate Assay, nature 2, 9.

13. KAM, E., and MA, S. (2011) Formulation and evaluation of silver nanoparticles as antibacterial and antifungal agents with a minimal cytotoxic effect, International Journal of Drug Delivery 3, 293-304.

14. Jain, P. K., Huang, X., El-Sayed, I. H., and El-Sayed, M. A. (2008) Noble metals on the nanoscale: optical and photothermal properties and some applications in imaging, sensing, biology, and medicine, Accounts of Chemical Research 41, 1578-1586. DOI: $10.1021 / \operatorname{ar} 7002804$ 
15. Eid, K. A., and Azzazy, H. M. (2012) Controlled synthesis and characterization of hollow flower-like silver nanostructures, International Journal of Nanomedicine 7, 1543. DOI: $10.2147 /$ IJN.S26524

16. Eid, K. A., and Azzazy. (2013) Sustained broad-spectrum antibacterial effects of nanoliposomes loaded with silver nanoparticles, Nanomedicine, Future Medicine 9,11. DOI: 10.2217/nnm.13.89

17. You, T., Niwa, O., Tomita, M., and Hirono, S. (2003) Characterization of platinum nanoparticle-embedded carbon film electrode and its detection of hydrogen peroxide, Analytical chemistry 75, 20802085. DOI: $10.1021 / \mathrm{ac} 026337 \mathrm{~W}$

18. Porcel, E., Liehn, S., Remita, H., Usami, N., Kobayashi, K., Furusawa, Y., Le Sech, C., and Lacombe, S. (2010) Platinum nanoparticles: a promising material for future cancer therapy?, Nanotechnology 21, 085103.

19. Hikosaka, K., Kim, J., Kajita, M., Kanayama, A., and Miyamoto, Y. (2008) Platinum nanoparticles have an activity similar to mitochondrial NADH: ubiquinone oxidoreductase, Colloids and Surfaces B: Biointerfaces 66, 195-200. DOI: 10.1016/j.colsurfb.2008.06.008

20. Barnham, K. J., Kenche, V. B., Ciccotosto, G. D., Smith, D. P., Tew, D. J., Liu, X., Perez, K., Cranston, G. A., Johanssen, T. J., and Volitakis, I. (2008) Platinum-based inhibitors of amyloid- $\beta$ as therapeutic agents for Alzheimer's disease, Proceedings of the National Academy of Sciences 105, 68136818.doi: 10.1073/0800712105

21. Park, E.-J., Kim, H., Kim, Y., and Park, K. (2010) Intratracheal instillation of platinum nanoparticles may induce inflammatory responses in mice, Archives of pharmacal research 33, 727-735. DOI 10.1007/s12272-010-0512-y

22. Yoshihisa, Y., Honda, A., Zhao, Q. L., Makino, T., Abe, R., Matsui, K., Shimizu, H., Miyamoto, Y., Kondo, T., and Shimizu, T. (2010) Protective effects of platinum nanoparticles against UV-light-induced epidermal inflammation, Experimental dermatology 19, 1000-1006. DOI: 10.1111/j.1600-0625.2010.01128.x

23. Wang, M. (2007) Materials selection and scaffold fabrication for tissue engineering in orthopaedics, In Advanced Bioimaging Technologies in Assessment of the Quality of Bone and Scaffold Materials, pp 259-288, Springer.

24. Subia, B., Kundu, J., and Kundu, S. (2010) Biomaterial scaffold fabrication techniques for potential tissue engineering applications, Tissue engineering, Intech web, Vienna, Austria, 141-157.

25. Mahapatro, A., Matos Negron, T. D., Bonner, C., and Abdel-Fattah, T. M. (2013) Nanolayers on Magnesium (Mg) Alloy for Metallic Bone Tissue Engineering Scaffolds, Journal of Biomaterials and Tissue Engineering 3, 196-204. DOI: 10.1016/j.biomaterials.2007.04.014

26. Wang, H., Li, Y., Zuo, Y., Li, J., Ma, S., and Cheng, L. (2007) Biocompatibility and osteogenesis of biomimetic nanohydroxyapatite/polyamide composite scaffolds for bone tissue engineering, Biomaterials 28, 3338-3348.

27. Gamal A Abdel Hakeam, Kamel Eid, et al., (2013) Laser Surface Modification of Poly ( $\varepsilon$-caprolactone) Scaffold for Artificial Skin Applications, Am. J. Biomed. Sci 5,1,113. 